\title{
BMJ Open Protocol for a scoping review on rehabilitation among individuals who experience homelessness and traumatic brain injury
}

\author{
Vincy Chan (D) , ${ }^{1,2,3}$ Maria Jennifer Estrella, ${ }^{4}$ Jessica Babineau, ${ }^{5,6}$ \\ Angela Colantonio ${ }^{1,2,3,4}$
}

To cite: Chan V, Estrella MJ, Babineau J, et al. Protocol for a scoping review on rehabilitation among individuals who experience homelessness and traumatic brain injury. BMJ Open 2021;11:e052942. doi:10.1136/ bmjopen-2021-052942

- Prepublication history and additional supplemental material for this paper are available online. To view these files, please visit the journal online (http://dx.doi.org/10.1136/ bmjopen-2021-052942).

Received 01 May 2021 Accepted 18 October 2021

Check for updates

(C) Author(s) (or their employer(s)) 2021. Re-use permitted under CC BY-NC. No commercial re-use. See rights and permissions. Published by BMJ.

For numbered affiliations see end of article.

Correspondence to

Dr Vincy Chan;

vincy.chan@uhn.ca

\section{ABSTRACT}

Introduction Rehabilitation is key to improving outcomes and quality of life after traumatic brain injury (TBI). However, individuals experiencing homelessness are rarely represented in research that informs evidencebased rehabilitation guidelines even though TBI is disproportionately prevalent among this population. This protocol is for a scoping review to explore the extent to which rehabilitation, including the types of rehabilitation interventions, is available to, or used by, individuals who experience homelessness and TBI to inform (1) opportunities to integrate rehabilitation for individuals experiencing homelessness and TBI, (2) considerations for existing clinical and practice guidelines for rehabilitation and (3) recommendations for future research.

Methods and analysis The scoping review will be guided by six stages described in scoping review methodology frameworks. Electronic databases (MEDLINE, Embase and Embase Classic, Cochrane CENTRAL Register of Clinical Trials, CINAHL, APA PsycINFO, Applied Social Sciences Index and Abstracts, and Nursing and Allied Health), reference list of included articles and scoping or systematic reviews identified from the search and grey literature, defined as reports from relevant brain injury, housing and rehabilitation organisations, will be searched. Two reviewers will independently screen all articles based on predetermined inclusion and exclusion criteria. A descriptive numerical summary of data items will be provided and qualitative content analytic techniques will be used to identify and report common themes. Preliminary findings will be shared with stakeholders to seek feedback on the implications of the results.

Ethics and dissemination Ethics review will not be required, as only publicly available data will be analysed. Findings from the scoping review will be published in a peer-reviewed journal and presented at scientific meetings and to stakeholders, defined as service providers in the housing and TBI sectors; health professionals who provide care for individuals with TBI and/or homelessness; health administrators, decisionmakers and policy-makers; researchers; and caregivers or family members of individuals with lived experience of TBI and homelessness.
Strengths and limitations of this study

To the best of our knowledge, this is the first scoping review of rehabilitation among individuals experiencing homelessness and traumatic brain injury (TBI); findings will inform (1) opportunities to integrate rehabilitation for individuals experiencing homelessness and TBI, (2) considerations for existing clinical and practice guidelines for rehabilitation and (3) recommendations for future research.

- This protocol is guided by scoping review methodology frameworks to improve methodological rigour, an identified limitation of existing rehabilitation scoping reviews, and describes a transparent approach to comprehensively identify literature on rehabilitation and TBI among underserved populations, which increases replicability.

- Intersecting sex, gender, social identities and vulnerabilities will be considered in the charting of the data, analysis and reporting of findings.

- We acknowledge the risk of publication bias because only peer-reviewed articles or published reports will be included.

- To evaluate and report on the risk of publication bias, non-English articles will not be immediately excluded; the decision to include or exclude non-English language full texts will be determined at the time of the review, taking into account the availability of resources and the proportion of non-English full-text articles.

\section{INTRODUCTION}

Homelessness is a global crisis affecting an estimated 100 million people worldwide and becoming increasingly prevalent in many countries. ${ }^{1}$ In Canada, more than 200000 individuals experience homelessness every year. ${ }^{2}$ This number is based on a 2016 national report; however, recent studies suggest that the state of homelessness in the country is worsening. ${ }^{3} 4$ Individuals who experience homelessness suffer from a broad range of health concerns, including systemic disorders 
(e.g., chronic obstructive pulmonary disease, seizures, arthritis, musculoskeletal disorders, tuberculosis) and mental health conditions and substance use, all contributing to high mortality rates. ${ }^{5-7}$ Consistently underrecognised is traumatic brain injury (TBI). Defined as 'an alteration in brain function or other evidence of brain pathology caused by an external force, ${ }^{8}$ TBI is a serious public health problem and the leading cause of death and disability among all trauma-related injuries globally. ${ }^{9}$

Approximately one in two individuals who are homeless or precariously housed experience a lifetime TBI while almost one in four individuals experience a moderate to severe TBI. ${ }^{10}$ Evidence suggests that the relationship between TBI and homelessness is bidirectional, with TBI preceding and prolonging homelessness. ${ }^{10-12}$ The first incidence of TBI was often found to occur before the onset of homelessness, ${ }^{12}$ and moderate to severe TBI was associated with the initial loss of stable housing and a longer duration of homelessness and precarious housing. ${ }^{11}$ Individuals with TBI often experience cognitive and behavioural challenges related to memory, attention, mental fatigue and irritability; fatigue and balance problems; and an increased likelihood of developing neurological and psychiatric conditions, all of which impact various areas of life and can be long-lasting or permanent. ${ }^{13-17}$ Among those experiencing homelessness specifically, TBI has been found to be associated with poorer physical and mental health status, increased likelihood of seizures, mental health and substance use problems, higher risk for suicide, increased health service use and increased criminal justice system involvement. ${ }^{10} 1819$ TBI-specific challenges, combined with other physical and mental health problems, and factors such as financial constraints and lack of social supports, lead to difficulties maintaining housing and increase the risk for homelessness. As the literature suggests, homelessness is a fluid experience characterised by frequent shifts in physical living situations (i.e., unsheltered, emergency and provisionally accommodated) and changes in housing status (i.e., at risk of homelessness to experiencing homelessness).$^{20}$ If unaddressed, the effects of TBI and homelessness combined could lead to a cycle of repeated TBIs, prolonged homelessness, and substantial economic and health-related costs. ${ }^{19} 2122$

The long-term negative outcomes of TBI, along with the intersecting challenges experienced by individuals who experience homelessness, demand long-term specialised supports including rehabilitation. Rehabilitation, defined as 'a set of interventions designed to optimise functioning and reduce disability in individuals with health conditions in interaction with their environment,' has been identified by WHO as an integral part of the healthcare continuum; when integrated with primary healthcare, rehabilitation can reduce disability, optimise the outcomes of other interventions and support full recovery. ${ }^{23}$ Over the years, various rehabilitation interventions have been found beneficial in managing TBI symptoms and facilitating outcomes such as community integration and quality of life. ${ }^{24} 25$ The growing evidence base on TBI rehabilitation has informed the development of evidence-based guidelines that provide comprehensive recommendations for TBI $\mathrm{care}^{26}$; however, the research informing these guidelines do not sufficiently, if at all, represent underserved populations, such as individuals experiencing homelessness. Further, while reviews on TBI rehabilitation or clinical guidelines for homelessness exist, ${ }^{2425}{ }^{27}$ there is none to date that focuses on rehabilitation interventions across disciplines for individuals who experience homelessness and TBI. This paucity of information suggests that existing evidence-based practice guidelines do not reflect rehabilitation that are specific to the needs of individuals who experience homelessness and TBI.

This protocol is for a scoping review that aims to address this gap, by exploring the extent to which rehabilitation, including types of rehabilitation interventions, is available to or used by individuals experiencing homelessness and TBI. This review will also aim to summarise findings across sex, gender and other identity factors (e.g., age, race, ethnicity and disability). To date, there is a lack of information across intersecting identities even though they contribute to unique experiences that cannot be addressed by looking at a single facet of identity. ${ }^{28-31}$ The results of the scoping review will inform (1) opportunities to integrate rehabilitation for individuals experiencing homelessness and TBI, (2) considerations for existing clinical and practice guidelines, and (3) recommendations for future research.

\section{METHOD AND ANALYSIS}

The scoping review will be guided by six stages described in Arksey and O'Malley's scoping review methodology framework and Levac et $a l$ 's additional recommendations to this framework ${ }^{32}{ }^{33}$ : (1) identifying the research question, (2) identifying relevant studies, (3) selecting studies, (4) charting the data, (5) collating, summarising, and reporting the results, and (6) consultation, which is an optional stage. The reporting of the scoping review will follow the Preferred Reporting Items for Systematic Reviews and Meta-Analyses extension for Scoping Reviews (PRISMA-ScR). ${ }^{34}$

\section{Stage 1: identifying the research question}

The research question is: 'To what extent is rehabilitation, including the types of rehabilitation intervention, available to, or used by, individuals experiencing homelessness and TBI?' As rehabilitation encompasses a variety of disciplines and homelessness a variety of living situations, the following parameters and definitions will guide the scoping review, including the search strategy, study selection, charting of data and reporting of findings.

Rehabilitation will be defined using (1) the WHO's definition $^{35}$ and (2) rehabilitation teams identified in evidence-based guidelines for TBI rehabilitation. ${ }^{2636}$ Homelessness will be defined using the Canadian 
Table 1 Parameters and associated definitions for rehabilitation and homelessness

\begin{tabular}{|c|c|c|}
\hline Concept & Parameter & Definition \\
\hline \multirow[t]{2}{*}{ Rehabilitation } & WHO's definition of rehabilitation ${ }^{35}$ & $\begin{array}{l}\text { 'A set of interventions designed to optimise functioning and } \\
\text { reduce disability in individuals with health conditions in interaction } \\
\text { with their environment' }\end{array}$ \\
\hline & $\begin{array}{l}\text { Healthcare providers/professional } \\
\text { disciplines identified in evidence- } \\
\text { based clinical practice guidelines } \\
\text { for TBI rehabilitation. }{ }^{26} 36\end{array}$ & $\begin{array}{l}\text { Speech-language pathologists } \\
\text { Occupational therapist } \\
\text { Physiotherapist } \\
\text { Social worker } \\
\text { Neuropsychologist and psychometrist } \\
\text { Psychologist with expertise in behavioural therapy } \\
\text { Nurse } \\
\text { Physician and/or physiatrist } \\
\text { Rehabilitation support personnel } \\
\text { Nutritionist }\end{array}$ \\
\hline \multirow{2}{*}{ Homelessness $^{20}$} & Emergency sheltered & $\begin{array}{l}\text { Individuals who cannot secure permanent housing and are } \\
\text { accessing shelters or other system supports: } \\
\text { 'Emergency overnight shelters for people who are } \\
\text { homelessness' or } \\
\text { 'Shelters for individuals/families impacted by family violence' } \\
\text { or } \\
\text { 'Emergency shelter for people fleeing a natural disaster or } \\
\text { destruction of accommodation due to fires, floods, etc.' }\end{array}$ \\
\hline & Provisionally accommodated & $\begin{array}{l}\text { Individuals without permanent shelter and are accessing } \\
\text { accommodations that offer no prospect of permanent: } \\
\text { Interim housing } \\
\text { Living temporality with others } \\
\text { Accessing short-term, temporary rental without security of } \\
\text { tenure } \\
\text { Living in institutional care and lack housing arrangements } \\
\text { Accommodation/reception centres for recently arrived } \\
\text { immigrants and refugees }\end{array}$ \\
\hline
\end{tabular}

TBI, Traumatic Brain Injury; WHO, World Health Organization.

Observatory of Homelessness (formerly the Canadian Homelessness Research Network) typology of homelessness that encompasses the following physical living situations at the time of the research study: (1) unsheltered, (2) emergency sheltered and (3) provisionally accommodated. ${ }^{20}$ Table 1 illustrates the parameters and associated definitions for rehabilitation and homelessness that will guide the review.

\section{Stage 2: identifying relevant studies}

The comprehensive database search strategy proposed in this protocol was developed with an information specialist (JB) and involved iterative revisions with research team members who possess research and subject-matter expertise relevant to rehabilitation, TBI and homelessness (see online supplemental file 1 ). The search strategy is developed for the MEDLINE ALL (in Ovid, including Epub Ahead of Print, In-Process \& Other Non-Indexed Citations, Ovid MEDLINE(R) Daily) database, and will be translated to: Embase and
Embase Classic (Ovid), Cochrane CENTRAL Register of Clinical Trials (Ovid), CINAHL (EBSCO), APA PsycINFO (Ovid), Applied Social Sciences Index and Abstracts (Proquest) and Nursing and Allied Health (Proquest).

The following concepts were developed to form the search strategy:

- Homelessness

- Rehabilitation

- TBI or cognitive impairment

The final search strategy structure, $(A+B)$ OR $(A+C)$, will be used to search each database. No language or date limits will be placed on search strategies. In addition to comprehensive and structured database searching, reference lists of included articles and scoping or systematic review articles identified from the search, and grey literature, defined as reports from relevant brain injury, housing and rehabilitation organisations will be searched (see online supplemental file 1). 


\section{Stage 3: study selection}

To be included in the scoping review, peer-reviewed articles, grey literature and reference lists of included primary research articles and scoping or systematic reviews must meet the following inclusion criteria: (1) describe and/or document rehabilitation and/or rehabilitation interventions that aim to optimise functioning and reduce disability in interaction with their environment or the delivery of care or describe and/or document rehabilitation services provided by healthcare providers/professional disciplines, as defined in table 1; (2) focus on individuals who are experiencing homelessness at the time of the research study, as defined in table 1; (3) include individuals with TBI and (4) report primary research findings.

Dissertations, conference proceedings and articles that are narrative, commentaries or describe a theory or framework without reporting primary research findings will be excluded. Articles that look at the broader brain-injured population (e.g., acquired brain injury) or individuals with cognitive impairment without specific mention of TBI will also be excluded.

Relevant studies retrieved using the above search strategy will be imported into EndNote X8.2 for reference management and Covidence for deduplication and study selection. ${ }^{37}$ Two reviewers will independently screen all articles based on the above inclusion and exclusion criteria. At the title and abstract screen, articles that do not explicitly mention cognitive impairment or TBI will be considered for the full-text screen to confirm the study include individuals with TBI. Prior to formal screening, pilot testing of 20 titles and abstracts will be conducted, until a minimum $80 \%$ agreement using the kappa statistic is achieved between the reviewers. At the full-text review, pilot testing of $10 \%$ of the full-text articles will be conducted until a minimum of $80 \%$ agreement is achieved between the reviewers. Non-English language abstracts will be assessed using the published English abstract and the decision to include these articles in the full-text articles will be determined at the time of the review, considering the availability of resources and proportion of non-English full-text articles. Any discrepancies during the study selection stage will be resolved by consensus or consultation with a third reviewer. The study selection process will be presented using the PRISMA flow chart. ${ }^{34}$

\section{Stage 4: charting the data}

Table 2 presents the charting table for the scoping review, which will be continually refined, as recommended by Levac et al's methodology framework. ${ }^{32}$ One reviewer will independently complete the charting table for each study and the completed table will then be independently peerreviewed by a second reviewer. Similar to the study selection stage, charting of the data will begin with a random sample of five articles until a minimum of $80 \%$ agreement is achieved between the reviewers. Discrepancies in charting the data will be resolved by consensus or in consultation with a third reviewer.

\section{Stage 5: collating, summarising and reporting the results}

As recommended by Levac et al's methodology framework, ${ }^{32}$ stage 5 will follow three distinct steps that may be refined further towards the end of the review, based on the content of the included articles:

1. Analysing the data-a descriptive numerical summary of study characteristics, study sample, rehabilitation and barriers, facilitators, and gaps will be provided and qualitative content analytic techniques will be applied to inform steps 2 (reporting results) and 3 (applying meaning to the results ${ }^{38}$; this method of data analysis is appropriate for this review, as it allows for the quantification of data (i.e., taking into account the frequency of similar codes) in themes or category development. ${ }^{39}$ Furthermore, we will assess the quality of the included studies using the Study Quality Assessment Tools designed by methodologists from the Research Triangle Institute International and the National Heart, Lung and Blood Institute of the National Institutes of Health. These tools aim to assess the internal validity of a study, including sources of bias, confounding, study power and other factors, ${ }^{40}$ to critically appraise different study designs. These quality assessments tools will be used to inform the process of applying meaning to the results, specified in step 3. No articles will be eliminated based on the quality assessment.

2. Reporting results -findings will be reported in relation to the research question (the extent to which rehabilitation, including the types of rehabilitation interventions, is available to, or used by, individuals experiencing homelessness and $\mathrm{TBI}$ ).

3. Applying meaning to the results-implications for (1) opportunities to integrate rehabilitation for individuals experiencing homelessness and TBI, (2) considerations for existing clinical and practice guidelines for rehabilitation and (3) recommendations for future research will be considered.

\section{Stage 6: consultation}

Preliminary findings from stage 5 will be shared with stakeholders of this scoping review to identify additional literature and seek feedback on the implications of the review on (1) opportunities to integrate rehabilitation for individuals experiencing homelessness and TBI, (2) considerations for existing clinical and practice guidelines for rehabilitation and (3) recommendations for future research. Stakeholders include front-line staff and service providers in the housing and brain injury sectors; health administrators, decision-makers and policy-makers; health professionals who provide care for individuals with TBI and/or individuals who experience homelessness; researchers and trainees who conduct research on rehabilitation, TBI and homelessness; and caregivers or family members of individuals with lived experience of TBI and/or homelessness. 
Table 2 Charting table

\section{Data item}

\section{Description}

\section{Study characteristics Author \\ Year of publication \\ Country of study \\ Type of article \\ Study design \\ Objective}

Study sample
TBI

Homelessness

Age

Sex/gender

Sociodemographic
Note if the article was a peer-reviewed publication or grey literature. Specify if the study was quantitative, qualitative or mixed methods and describe the study design.

Describe the stated objective of the study.

Specify the definition of TBI or how TBI was identified/determined.

Specify the injury severity, time since injury, method of diagnosis/ screening, timing of TBI relative to homelessness (e.g., whether TBI predated homelessness, if the individual was homeless at the time of $\mathrm{TBI})$, and the sample (N, \%) of individuals with TBI.

Specify the definition of homelessness.

Specify the sample (N, \%) of individuals experiencing homelessness. Specify participants' age at the time of the study, at the time of TBI, and at the time of homelessness.

Specify if Sex-Based and Gender-Based Analysis Plus was considered in the study design. ${ }^{43}$

Note if and/or how sex and gender were defined in the study.

Specify the participants' sex and/or gender (N, \%).

Specify sociodemographic characteristics of the sample (e.g., race, ethnicity, religion, disability, geography, culture, income, education), including experiences consistent with those at risk of homelessness as defined in the $\mathrm{COH} .{ }^{20}$

Note if/describe how the sample of individuals with homelessness and $\mathrm{TBI}$ intersect with the criminal justice/legal system or experienced violence, including intimate partner violence.

Note if/describe how the article acknowledged and/or accounted for intersecting social identities and/or vulnerabilities. 
Table 2 Continued

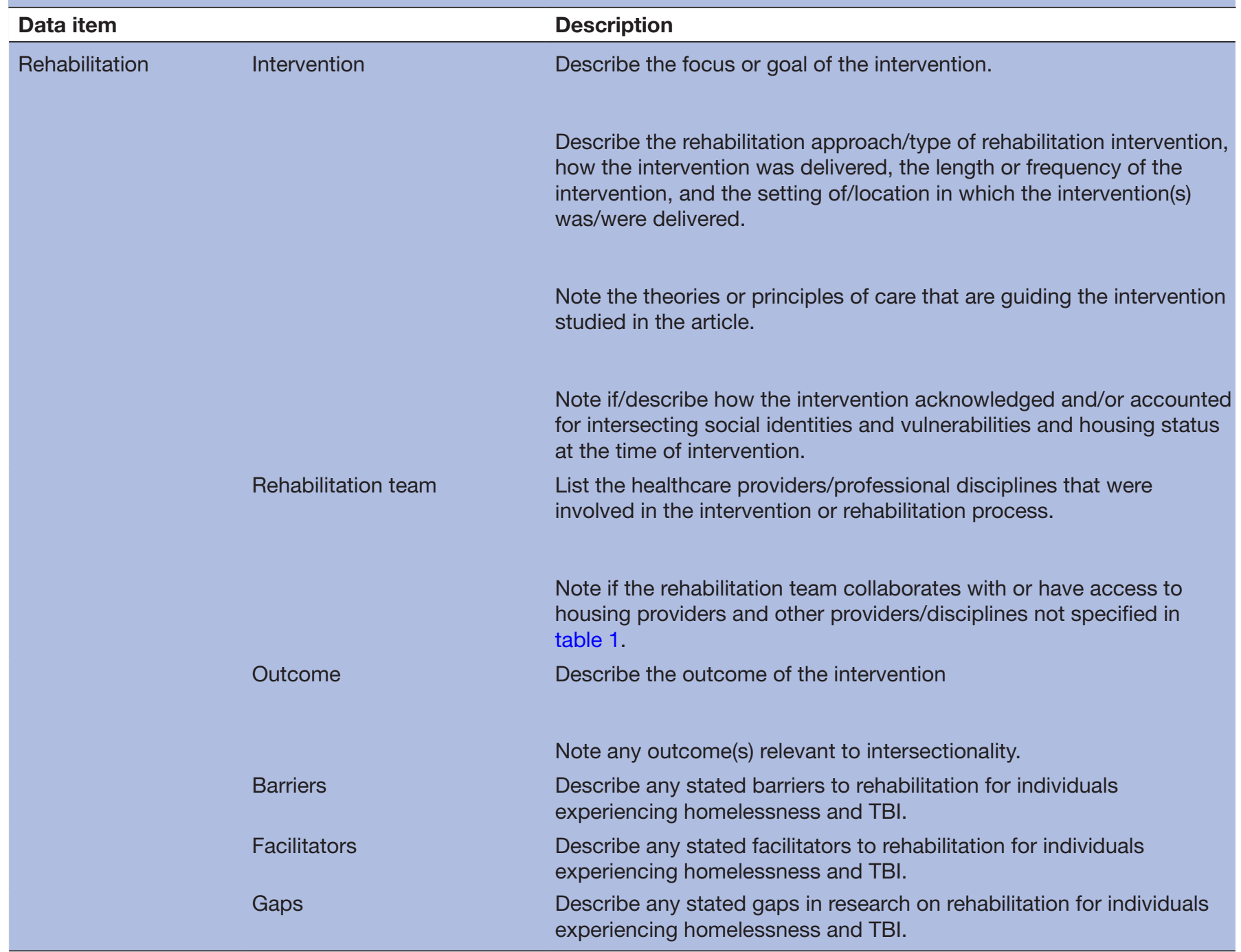

$\mathrm{COH}$, Canadian Observatory of Homelessness; TBI, Traumatic Brain Injury.

\section{Patient and public involvement}

Patients and the public were not involved in the creation of this scoping review protocol. However, stage 6 of our proposed methods will engage stakeholders of this scoping review.

\section{Ethics and dissemination}

Ethics review will not be required because only published and publicly available data will be analysed. The scoping review will be published in a peer-reviewed journal. Findings will be presented at scientific conferences and stakeholders defined in stage six of the scoping review.

\section{Strengths and limitations}

We acknowledge the risk of publication bias, as only peer-reviewed articles or published reports will be included. For example, pilot studies conducted as theses will not be captured in the scoping review unless they are published in peer-reviewed journals. However, this review will aim to minimise publication bias by including non-English articles in the title and abstract screen using the published English version of the abstract. The decision to include or exclude nonEnglish language full texts will be determined at the time of the review, taking into account the availability of resources and proportion of non-English full-text articles. Furthermore, it is recognised that community organisations serving individuals experiencing homelessness and/or TBI may produce non-peer-reviewed reports of the services they offer. As such, grey literature, defined as reports published by brain injury, housing, and rehabilitation organisations, will also be searched. We also acknowledge that the inclusion of quality assessment deviates from the methodology frameworks used to inform this protocol..$^{32} 33$ No studies will be excluded from this scoping review based on the quality assessment, however, results from the assessment will be considered when we apply meaning to the findings that are used to inform considerations for future research, the integration of rehabilitation, 
and clinical and best practices guidelines. Finally, this scoping review will not explicitly search for articles that only focus on individuals at risk of homelessness, defined as 'people who are not homeless, but whose current economic and/or housing situation is precarious or does not meet public health and safety standard. ${ }^{20}$ For example, articles that focus on precariously employed individuals without explicit mention of being unsheltered, emergency sheltered, and provisionally accommodated will not be included in this review. We acknowledge that homelessness is a fluid experience, with similar factors associated with unmet healthcare needs among those who are homeless and vulnerably housed. ${ }^{41}$ However, the goal of this scoping review is to explore the extent to which rehabilitation, including the types of rehabilitation intervention, is available to, or used by individuals experiencing homelessness and TBI. Rehabilitation studies focused on individuals with TBI who are unsheltered, emergency sheltered and provisionally accommodated at the time of the research study may describe a different rehabilitation experience than studies that focus on individuals at risk of homelessness or individuals with lived experience of homelessness who are in permanent housing. As such, this scoping review will not explicitly search for articles that only include individuals at risk of homelessness or who are currently in permanent housing. Instead, the charting and analysis of the data will identify and contextualise social determinants of health and other factors that put them at imminent risk of homelessness. It will also extract the definition of homelessness from the research study and note the location of the intervention(s). Future reviews on rehabilitation that are focused specifically on individuals at risk of homelessness, or specific populations at risk of homelessness, as well as studies focused on individuals with lived experience of homelessness and are in permanent housing are encouraged.

To the best of our knowledge, this is the first protocol for a scoping review on rehabilitation among individuals experiencing homelessness and TBI. This protocol is guided by scoping review methodology frameworks to improve methodological rigour, which also addresses an identified limitation of existing rehabilitation scoping review. ${ }^{42}$ This protocol also describes a transparent approach to comprehensively identify literature on rehabilitation and TBI among underserved populations, which increases credibility and replicability. Importantly, intersecting sex, gender, social identities and vulnerabilities, which are often overlooked in the literature, will be considered in the charting of the data, analysis and reporting of findings. Findings from the scoping review will provide an evidence-based foundation to inform (1) opportunities to integrate rehabilitation for TBI for individuals experiencing homelessness, (2) considerations for existing clinical and practice guidelines, and (3) recommendations future research.
Author affiliations

${ }^{1} \mathrm{KITE}$, Toronto Rehabilitation Institute-University Health Network, Toronto, Ontario, Canada

${ }^{2}$ Rehabilitation Sciences Institute, University of Toronto, Toronto, Ontario, Canada ${ }^{3}$ Institute of Health Policy, Management and Evaluation, University of Toronto, Toronto, Ontario, Canada

${ }^{4}$ Department of Occupational Science \& 0ccupational Therapy, University of Toronto, Toronto, Ontario, Canada

${ }^{5}$ Library \& Information Services, University Health Network, Toronto, Ontario, Canada

${ }^{6}$ The Institute for Education Research, University Health Network, Toronto, Ontario, Canada

Acknowledgements The authors would like to thank Ms. Samira Omar, PhD Candidate at the Rehabilitation Sciences Institute, University of Toronto, for reviewing the final manuscript and providing editorial feedback.

Contributors VC and AC conceptualised the study. VC, MJE and JB developed the search strategy. VC and MJE formulated the design and drafted the manuscript. All authors critically reviewed the manuscript and approved the final manuscript.

Funding This research has been funded in part by the Canada Research Chairs Program (Fund Number N/A) and the Ontario Ministry of Health and Long-Term Care, Ministry Grant \#725A. The views expressed in the publication are the views of the authors and do not necessarily reflect those of the Ministry of Health and Long-Term Care.

Competing interests None declared.

Patient consent for publication Not applicable.

Provenance and peer review Not commissioned; externally peer reviewed.

Supplemental material This content has been supplied by the author(s). It has not been vetted by BMJ Publishing Group Limited (BMJ) and may not have been peer-reviewed. Any opinions or recommendations discussed are solely those of the author(s) and are not endorsed by BMJ. BMJ disclaims all liability and responsibility arising from any reliance placed on the content. Where the content includes any translated material, BMJ does not warrant the accuracy and reliability of the translations (including but not limited to local regulations, clinical guidelines, terminology, drug names and drug dosages), and is not responsible for any error and/or omissions arising from translation and adaptation or otherwise.

Open access This is an open access article distributed in accordance with the Creative Commons Attribution Non Commercial (CC BY-NC 4.0) license, which permits others to distribute, remix, adapt, build upon this work non-commercially, and license their derivative works on different terms, provided the original work is properly cited, appropriate credit is given, any changes made indicated, and the use is non-commercial. See: http://creativecommons.org/licenses/by-nc/4.0/.

ORCID iD

Vincy Chan http://orcid.org/0000-0002-6884-044X

\section{REFERENCES}

1 Affordable housing, inclusive economic policies key to ending homelessness, speakers say as social development Commission begins annual session, 2020. United nations meetings coverage and press releases.United Nations. Available: https://www.un.org/press/ en/2020/soc4884.doc.htm [Accessed October 26, 2021].

2 Gaetz S, Dej E, Richter T. The state of homelessness in Canada 2016 Toronto: Canadian Observatory on Homelessness Press., 2016. ISBN: 978-1-77221-057-6. https://www.homelesshub.ca/SOHC2016

3 Strobel S, Burcul I, Dai JH, et al. Characterizing people experiencing homelessness and trends in homelessness using population-level emergency department visit data in Ontario, Canada. Health Rep 2021;32:13-23.

4 Canadian Alliance to End Homelessness 2020-1698. One in five Canadians have a friend or acquaintance who has been homeless; majority support building new affordable housing; 2020. https:// d3n8a8pro7vhmx.cloudfront.net/caeh/pages/81/attachments/ original/1597194371/CAEH-Nanos_Poll_Report_-_Housing Homelessness_\%281\%29.pdf?1597194371 [Accessed 26 Oct 2021].

5 Hwang SW. Homelessness and health. CMAJ 2001;164:229-33.

6 Frankish CJ, Hwang SW, Quantz D. Homelessness and health in Canada: research lessons and priorities. Can J Public Health 2005;96 Suppl 2:S23-9. 
7 Aldridge RW, Story A, Hwang SW, et al. Morbidity and mortality in homeless individuals, prisoners, sex workers, and individuals with substance use disorders in high-income countries: A systematic review and meta-analysis. Lancet 2018;391:241-50.

8 Menon DK, Schwab K, Wright DW, et al. Position statement: Definition of traumatic brain injury. Arch Phys Med Rehabil 2010;91:1637-40.

9 Rubiano AM, Carney N, Chesnut R, et al. Global neurotrauma research challenges and opportunities. Nature 2015;527:S193-7.

10 Stubbs JL, Thornton AE, Sevick JM, et al. Traumatic brain injury in homeless and marginally housed individuals: A systematic review and meta-analysis. Lancet Public Health 2020;5:e19-32.

11 Stubbs JL, Thornton AE, Gicas KM, et al. Characterizing traumatic brain injury and its association with losing stable housing in a community-based sample: Caractérisation d'une lésion cérébrale traumatique et de son association avec la perte d'un logement stable dans un échantillon communautaire. Canadian journal of psychiatry Revue canadienne de psychiatrie $2021 ; 7067437211000665$

12 Topolovec-Vranic J, Ennis N, Colantonio A, et al. Traumatic brain injury among people who are homeless: A systematic review. BMC Public Health 2012;12:1059.

13 Ponsford JL, Downing MG, Olver J, et al. Longitudinal follow-up of patients with traumatic brain injury: Outcome at two, five, and ten years post-injury. J Neurotrauma 2014;31:64-77.

14 Ruet A, Bayen E, Jourdan C, et al. A detailed overview of long-term outcomes in severe traumatic brain injury eight years post-injury. Front Neurol 2019;10:120.

15 Ahman S, Saveman B-I, Styrke J, et al. Long-Term follow-up of patients with mild traumatic brain injury: A mixed-method study. $J$ Rehabil Med 2013;45:758-64.

16 Perry DC, Sturm VE, Peterson MJ, et al. Association of traumatic brain injury with subsequent neurological and psychiatric disease: A meta-analysis. J Neurosurg 2016;124:511-26.

17 Scholten AC, Haagsma JA, Cnossen MC, et al. Prevalence of and risk factors for anxiety and depressive disorders after traumatic brain injury: A systematic review. J Neurotrauma 2016;33:1969-94.

18 Hwang SW, Colantonio A, Chiu S, et al. The effect of traumatic brain injury on the health of homeless people. CMAJ 2008;179:779-84.

19 To MJ, O’Brien K, Palepu A, et al. Healthcare utilization, legal incidents, and victimization following traumatic brain injury in homeless and Vulnerably housed individuals: A prospective cohort study. J Head Trauma Rehabil 2015;30:270-6.

20 Gaetz S, Barr C, Friesen A, Harris B, Hill C, Kovacs-Burns K, Kovacs-Burns K, Pearce B, Turner A, Marsolais A. Canadian definition of homelessness. Toronto Canadian Observatory on Homelessness Press; 2012. https://www.homelesshub.ca/sites/ default/files/COHhomelessdefinition.pdf [Accessed October 26, 2021].

21 Svoboda T, Ramsay JT. High rates of head injury among homeless and low-income housed men: A retrospective cohort study. Emerg Med J 2014;31:571-5.

22 Adshead CD, Norman A, Holloway M. The inter-relationship between acquired brain injury, substance use and homelessness the impact of adverse childhood experiences: An interpretative phenomenological analysis study. Disabil Rehabil 2021;43:1-13.

23 World Health Organization. Access to rehabilitation in primary health care: An ongoing challenge. Contract No.: WHO/HIS/SDS/2018.40. Geneva: World Health Organization, 2018.
24 Gordon WA, Zafonte R, Cicerone K, et al. Traumatic brain injury rehabilitation: State of the science. Am J Phys Med Rehabil 2006;85:343-82.

25 Teasell R, Marshall S, Cullen N. Evidence-Based review of moderate to severe acquired brain injury (ERABI) version 13. Toronto, ON: Ontario Neurotrauma Foundation, 2019.

26 Bayley M, Swaine B, Lamontagne ME, et al. INESSS-ONF clinical practice guideline for the rehabilitation of adults with moderate to severe traumatic brain injury, 2017Ontario Neurotrauma Foundation. Available: https://braininjuryguidelines.org/modtosevere/ [Accessed October 26, 2021].

27 Pottie K, Kendall CE, Aubry T, et al. Clinical guideline for homeless and vulnerably housed people, and people with lived homelessness experience. CMAJ 2020;192:E240.

28 Markowitz FE, Syverson J, Race SJ. Race, gender, and homelessness stigma: effects of perceived Blameworthiness and dangerousness. Deviant Behav 2021;42:919-31.

29 Weisz C, Quinn DM. Stigmatized identities, psychological distress, and physical health: Intersections of homelessness and race. Stigma Health 2018;3:229-40.

30 Whitzman C. At the Intersection of Invisibilities: Canadian women, homelessness and health outside the 'big city'. Gender, Place \& Culture 2006;13:383-99.

31 Colantonio A. Sex, gender, and traumatic brain injury: a commentary Arch Phys Med Rehabil 2016;97:S1-4.

32 Levac D, Colquhoun H, O'Brien KK. Scoping studies: Advancing the methodology. Implement Sci 2010;5:69.

33 Arksey H, O'Malley L. Scoping studies: Towards a methodological framework. Int J Soc Res Methodol 2005;8:19-32.

34 Tricco AC, Lillie E, Zarin W, et al. PRISMA extension for scoping reviews (PRISMA-ScR): Checklist and explanation. Ann Intern Med 2018;169:467-73.

35 World Health Organization. Rehabilitation, 2021. Available: https:// www.who.int/news-room/fact-sheets/detail/rehabilitation

36 Reed N, Zemek R, Dawson J. Living guideline for diagnosing and managing pediatric concussion, 2021. Available: https:// pedsconcussion.com/

37 Covidence systematic review software.Melbourne, Australia, 2021.Veritas Health Innovation. Available: https://www.covidence. org/

38 Hsieh H-F, Shannon SE. Three approaches to qualitative content analysis. Qual Health Res 2005;15:1277-88.

39 Vaismoradi M, Snelgrove S. Theme in qualitative content analysis and thematic analysis. Forum: Qualitative Social Research 2019;20:1-14.

40 National Heart, Lung, and Blood Institute. Background: Development and use of study quality assessment tools. Available: http://nhlbi.nih. gov/node/80102 [Accessed October 26, 2021].

41 Argintaru N, Chambers C, Gogosis E, et al. A cross-sectional observational study of unmet health needs among homeless and vulnerably housed adults in three Canadian cities. BMC Public Health 2013;13:577.

42 Colquhoun HL, Jesus TS, O'Brien KK, et al. Scoping review on rehabilitation scoping reviews. Arch Phys Med Rehabil 2020;101:1462-9.

43 Canada WaGE. What is gender-based analysis plus, 2021.Government of Canada. Available: https://women-genderequality.canada.ca/en/gender-based-analysis-plus/what-genderbased-analysis-plus.html 